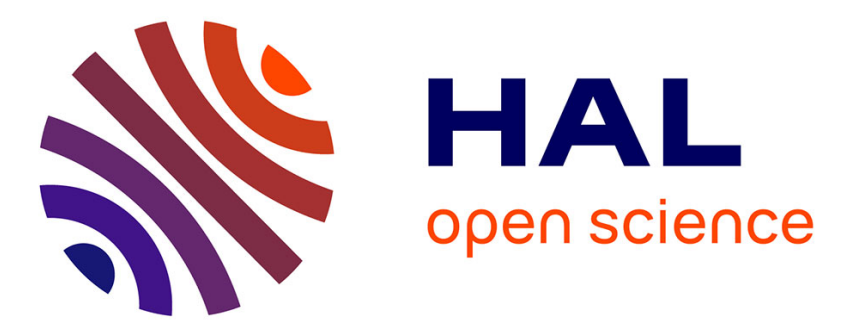

\title{
Daily oscillation of odorant detection in rat olfactory epithelium
}

\author{
Adrien François, Vincent Bombail, David D. Jarriault, Adrien Acquistapace, \\ Denise Grebert, Xavier Grosmaitre, Nicolas Meunier
}

\section{To cite this version:}

Adrien François, Vincent Bombail, David D. Jarriault, Adrien Acquistapace, Denise Grebert, et al.. Daily oscillation of odorant detection in rat olfactory epithelium. European Journal of Neuroscience, 2017, 45 (12), pp.1613-1622. 10.1111/ejn.13600 . hal-01549617

\section{HAL Id: hal-01549617 https://hal.science/hal-01549617}

Submitted on 28 Jun 2017

HAL is a multi-disciplinary open access archive for the deposit and dissemination of scientific research documents, whether they are published or not. The documents may come from teaching and research institutions in France or abroad, or from public or private research centers.
L'archive ouverte pluridisciplinaire HAL, est destinée au dépôt et à la diffusion de documents scientifiques de niveau recherche, publiés ou non, émanant des établissements d'enseignement et de recherche français ou étrangers, des laboratoires publics ou privés.

\section{다(1)(2)}

Distributed under a Creative Commons Attribution - ShareAlikel 4.0 International 


\title{
Daily oscillation of odorant detection in rat olfactory epithelium
}

\author{
Adrien Francois, ${ }^{1,2}$ Vincent Bombail, ${ }^{1}$ David Jarriault, ${ }^{3}$ Adrien Acquistapace, ${ }^{1}$ Denise Grebert, ${ }^{1}$ \\ Xavier Grosmaitre ${ }^{3}$ (D) and Nicolas Meunier, ${ }^{1,2}$ (D) \\ ${ }^{1}$ INRA, UR1197 NeuroBiologie de I'Olfaction, F-78350 Jouy-en-Josas, France \\ ${ }^{2} \mathrm{NBO}$, UVSQ, INRA, Université Paris-Saclay, Jouy-en-Josas, France \\ ${ }^{3}$ Centre des Sciences du Goût et de I'Alimentation, CNRS, INRA, Univ. Bourgogne Franche-Comté, Dijon, France
}

Keywords: biological rhythms, circadian rhythms, olfaction, sensory physiology, signal transduction

Edited by Giovanni Galizia

Received 19 December 2016, revised 20 April 2017, accepted 21 April 2017

\begin{abstract}
Most of biological variables follow a daily rhythm. It holds true as well for sensory capacities as two decades of research have demonstrated that the odorant induced activity in the olfactory bulbs oscillates during the day. Olfactory bulbs are the first central nervous system structures, which receive inputs from the olfactory neurons located in the nose olfactory epithelium in vertebrates. So far, data on variation in odorant detection in the olfactory epithelium throughout the day are missing. Using electroolfactogram recordings in rats housed under daily light and dark cycles, we found that the olfactory epithelium responsiveness varies during the day with a maximum in the beginning of the light phase. This fluctuation was consistent with cycling of transduction pathway gene expression in the olfactory epithelium examined by qPCR. It was also consistent with the levels of two transduction pathway proteins (olfactory-type G protein and adenylyl cyclase III) examined by western blot. Daily variations were also observed at the level of olfactory sensory neurons responses recorded by patch-clamp. To rule out a potential effect of the feeding status of the animal, we examined the variation in odorant response in starved animals during the day. We observed a similar pattern to ad libidum fed animals. Taken together, our results reveal that the olfactory epithelium sensitivity varies during the day in part due to modulation of the very first step of odorant detection.
\end{abstract}

\section{Introduction}

In mammals, many physiological and behavioural processes, such as sleep/wake and feeding/fasting cycles, follow a daily rhythm. Since the early seventies, those cycles have been attributed to a master circadian pacemaker following environmental cues localised in the suprachiasmatic nucleus (SCN). In addition, over the past decade, investigations have demonstrated the existence of peripheral oscillators that sustain local circadian rhythms (Dibner et al., 2010) including the olfactory bulb (OB). This neuronal structure receives inputs from the olfactory epithelium (OE), where odorant detection takes place through olfactory sensory neurons (OSN) which are in direct contact with the environment. Olfactory sensitivity has been shown to follow a circadian rhythm. It requires clock genes (GranadosFuentes et al., 2011) as well as the vasoactive intestinal polypeptide (Miller et al., 2014). OB responses to olfactory stimulations were shown to be higher during the dark phase (Amir et al., 1999; Funk

Correspondence: Dr N. Meunier, as above.

E-mail: nicolas.meunier@inra.fr

The associated peer review process communications can be found in the online version of this article.
\& Amir, 2000). A spontaneous c-Fos rhythm in the mitral and granular layer of $\mathrm{OB}$ has also been described (Granados-Fuentes et al., 2006). Finally, OB explants retain daily oscillations of Perl gene activity even from SCN-lesioned rats and cultured OB neurons display a circadian firing rate pattern (Granados-Fuentes et al., 2004). Although Per2 expression rhythmicity is altered by SCN lesion in vivo after several days (Ono et al., 2015), all those results clearly point out the $\mathrm{OB}$ as a peripheral oscillator, involved in driving olfactory circadian rhythm. The OE could be implicated in olfactory daily oscillations as well since its cells exhibit a circadian rhythm in vitro (Miller et al., 2014). Recently, evidence of in vivo variation in the mouse OE through the day has been reported with a demonstration of circadian variation in molecular PER2 expression (Honma et al., 2015) as well as an extensive study of molecular oscillation of gene expression in the OE (Saleh et al., 2015).

Here, using rats, we provide the first study of potential daily oscillation of OE sensitivity to odorant. OSN recorded by electroolfactogram (EOG) and patch-clamp showed daily variation in response amplitude. These variations were correlated with the transcript levels for olfactory transduction genes as well as to their protein levels. 


\section{Material and methods}

\section{Ethical approval}

All animal experiments were approved by the local ethics committee (COMETHEA; Avis 14/38) and conducted in accordance with the European guidelines for the care and use of laboratory animals (2010/63/EU). Wistar male rats (initially bought from Janvier Labs) were bred and housed at our local animal care facility with free access to water and food (M25 Extralabo, Dietex France). All experiments were performed on 5-week old male rats born from multiparous mothers and kept in pairs from weaning in a $12 \mathrm{~L}: 12 \mathrm{D}$ lighting schedule (lights on at 8:00). For EOG and qPCR experiments, we investigated four Zeitgeber times (ZT): ZT4, 10, 16 and 22 (lights off at ZT12). At each sampling time, six rats were gently manipulated during $5 \mathrm{~min}$ before being weighed then anesthetised (sodium pentobarbital, $60 \mathrm{mg} / \mathrm{kg}$ i.p.). They were decapitated $10 \mathrm{~min}$ later (one rat every $30 \mathrm{~min}$, starting from $1 \mathrm{~h} 30$ prior to ZT time point). The head was cut longitudinally, one hemi-head was used for EOG recordings, and the other was used for olfactory mucosa and bulb dissection. Livers were also collected and weighed. For western blot and cFos analysis, a further 12 rats were sampled at ZT4 and ZT22. For patch-clamp experiments, we used 18 rats at ZT4 and 17 rats at ZT22. To investigate the impact of metabolic signals on OE function, we used 24 rats which food was withdrawn $16 \mathrm{~h}$ prior to EOG recordings. Food restriction took place at ZT12, 18,24 and 6 (four groups of six rats for each time point) and we recorded EOG responses $16 \mathrm{~h}$ later at ZT4, 10, 16 and 22 respectively.

\section{Electroolfactogram recordings (EOG)}

To evaluate the global responses of OSNs at different times of day, EOG recordings in the olfactory mucosa were made from an opened nasal cavity configuration as described previously (Francois et al., 2013). The hemi-head was kept under a constant flow of humidified filtered air $(\sim 1000 \mathrm{~mL} / \mathrm{min})$ delivered through a $9 \mathrm{~mm}$ glass tube This tube was positioned $2 \mathrm{~cm}$ from the epithelial surface. Odour stimulations were performed by blowing air puffs $(200 \mathrm{~ms}, 200 \mathrm{~mL} /$ min) through an exchangeable Pasteur pipette enclosed in the glass tube containing a filter paper impregnated with $20 \mu \mathrm{L}$ of odorant diluted in mineral oil (Sigma Aldrich, Saint-Quentin Fallavier, France). The air flow difference between the constant flow and the odour driving flow minimises mechanical responses and odorant-free air stimulation (with mineral oil), always produced signals around $1 \mathrm{mV}$ amplitude. We used hexanal diluted from 1:10000 to $1: 10$ and limonene from $1: 1000$ to $1: 10$ as the latter has a lower vapour pressure value $(1.4 \mathrm{kPa}$ for hexanal vs. $0.19 \mathrm{kPa}$ for limonene at $25{ }^{\circ} \mathrm{C}$ ). We choose those two odorants because they are chemically very different (non-ramified saturated aldehyde vs. cyclic unsaturated terpene) and therefore act upon a wide range of olfactory receptors.

Electroolfactogram voltage signals were recorded using an XtraCell 2 channels amplifier (DIPSI, Chatillon, FRANCE) used in a DC current-clamp configuration $(I=0)$, low-pass bessel filtered at $1 \mathrm{KHz}$ and digitised at a rate of $2 \mathrm{kHz}$ using an Digidata 1322a A/D converter (Axon Instruments, Molecular Devices, Union City, $\mathrm{CA}$, USA). A reference $\mathrm{Ag} / \mathrm{AgCl}$ electrode was placed in the olfactory bulb. Recordings were made with glass micropipettes of $4-5 \mathrm{M} \Omega$ filled with a saline solution. EOG were recorded from the centre of turbinates IIb and III (central bones of the nasal cavity covered by olfactory epithelium). From these positions robust, reproducible and long-lasting EOG recordings can be made. To test for loss of signal, each EOG recordings session started with a control odorant $[(-)$ carvone diluted $1 / 100$ in mineral oil] which was used again at the end of the session. We did not observe any significant loss of signal for all rats used. Analyses were performed using Clampfit 9.2 (Axon Instruments) to measure the peak amplitude.

\section{Patch-clamp recordings}

Patch-clamp recordings of OSNs dendritic knobs were performed as described earlier (Grosmaitre et al., 2007; Cadiou et al., 2014; Jarriault \& Grosmaitre, 2015). To assess the total capacity of the OSNs' transduction pathway, we recorded the response to a mixture of IBMX and Forskolin diluted in Ringer or with Ringer alone. IBMX 2 a potent inhibitor of phosphodiesterase (Firestein et al., 1991; 5 we \& Gold, 1993), and Forskolin is an activator of adenylyl cyclase (Sklar et al., 1986). Solutions containing $100 \mu \mathrm{M}$ of IBMX and $10 \mu \mathrm{M}$ of Forskolin were prepared before each experiment by adding Ringer in DMSO stock solution. All chemicals were obtained from Sigma-Aldrich.

The junction potential was $\sim 9 \mathrm{mV}$ and was corrected in all experiments off-line. For Ringer and IBMX + Forskolin-induced transduction currents, signals were sampled at $5 \mathrm{kHz}$. Under voltageclamp mode, the signals were initially filtered at $10 \mathrm{kHz}$ and then at $2.9 \mathrm{kHz}$; under current-clamp mode, signals were filtered at $2.9 \mathrm{kHz}$. Action potential firing was recorded in current-clamp mode at $10 \mathrm{kHz}$ sampling frequency for spontaneous activity recordings and $50 \mathrm{kHz}$ for current injection recordings.

Data were analysed using custom-made routines written in Matlab (The MathWorks, Natick, MA, USA). We measured the following characteristics of the responses to odorants in voltage clamp: maximum amplitude, rise time (duration in $\mathrm{ms}$ from 10 to $90 \%$ of the maximum amplitude), time at $50 \%$ (duration in $\mathrm{ms}$ of the response width at $50 \%$ of the maximum amplitude), total current elicited (area under the curve in pAs). In current clamp, firing frequency was analysed using custom-made routines written in Matlab.

\section{Quantitative RT-Polymerase Chain Reaction (qPCR)}

Total RNA was extracted using the Trizol method from frozen olfactory epithelia or bulb (Francois et al., 2013) then treated with DNase I. OligodT first strand cDNA were synthesised from $2 \mu \mathrm{g}$ total RNA using superscript II reverse transcriptase (Invitrogen, Saint-Aubin, France) following the manufacturer recommendations. For quantitative PCR, $5 \mu \mathrm{L}$ of 125 fold diluted cDNA templates were added to the $15 \mu$ L-reaction mixture containing SYBR Green GoTaq ${ }^{\circledR}$ qPCR Master Mix (Promega, Charbonnieres, France) and 300 nм primers (F Forward/R Reverse: $\beta$-tubulin III F: 5'-TGAGGCCTCCTCTCA CAAGT-3', R: 5'-GGCCTGAATAGGTGTCCAAA-3', I7 F: 5'-CT CCCACCTCACTGTTGTGAT-3', R: 5'-GCGTAGAGTACAGAGA CCAGCTT-3', U131 F: 5'-TGGCAAGTAAAATTTGGTGCTA-3', R: $5^{\prime}$-CCAGTCAGAATGAACTCAGGAA-3', G $_{\text {olf }}$ F: 5'-GCATCTG GAATAACAGGTGGTT-3', R: 5'-GGCATTACTCCG GGAAATA GTCT-3', ACIII F: 5'-TTGACTCTCTCCTGGACAATCC-3', R: 5'-CTTGTAAAGCCATTGGTGTTGA-3', Cnga2 F: 5'-GCAAAAT GGGCAATCGACGTACTG-3', R: 5'-ACCTCCATACTAGCTGCC ACTTCA-3', PDE1C2 F: 5'-GGCTGCCATCCA TGACTATGAA CA-3', R: 5'-TTCGAAACTCCCTCCAGTCATCCT-3'). The qPCR was performed on a Mastercycler ${ }^{\circledR}$ ep realplex (Eppendorf) during 40 amplification cycles consisting of $45 \mathrm{~s}$ at $94{ }^{\circ} \mathrm{C}, 45 \mathrm{~s}$ at $60{ }^{\circ} \mathrm{C}$ and $45 \mathrm{~s}$ at $72{ }^{\circ} \mathrm{C}$. Quantification was achieved using the $\Delta \Delta C_{\mathrm{t}}$ method 
and mRNA expression was normalised to the expression level of the OSN specific $\beta$-tubulin III (Khan et al., 2011). Efficiency corrective factor was applied for each primer pair.

\section{Western blot}

For protein extraction, cells were lysed, homogenised in buffer (200 mм Tris- $\mathrm{HCl}$ pH7.4, 1.5 м NaCl, 2 mм EDTA, 2 mм EGTA, $1 \%$ NP40 and $1 \times$ anti-protease cocktail (Complete by Roche Diagnostics, Meylan, France). Protein concentrations were determined with a BCA assay (Pierce BCA Protein Assay Kit, 23225; Fisher Scientific, Illkirch, France). Samples were run on 10 or $12 \%$ denaturing SDS polyacrylamide gel electrophoresis and transferred to PVDF membranes. The membranes were incubated with primary antibodies in PBS/1\% milk powder, then incubated with a matching peroxidase-conjugated secondary antibody and proteins of interest were detected with an ECL blotting detection kit (Amersham Biosciences, Orsay, France). Mouse monoclonal $\beta$-actin antibody (A5441; 1:15 000; Sigma Aldrich), secondary sheep anti mouse (A5906; 1:10 000; Sigma Aldrich), rabbit polyclonal $\mathrm{G}_{\text {olf }}$ antibody (sc-383; 1:2000; Santa Cruz Biotechnology, Dallas, TX, USA), secondary goat anti rabbit (A0545; 1:5000; Sigma Aldrich), rabbit polyclonal ACIII antibody (sc-588; 1:300; Santa Cruz Biotechnology) and secondary goat anti rabbit (A0545; 1:5000; Sigma Aldrich) were used. Membranes were scanned (Fujifilm LAS-1000 luminescent image analyser), and the band intensity measured and analysed by densitometry with IMAGEJ (Rasband,W.S., ImageJ, U.S. National Institutes of Health, Bethesda, Maryland, USA, http://imagej.nih. gov/ij/).

\section{cFos immunohistochemistry}

Rats were exposed to almond oil (Fisher Scientific) diluted 1:100 in mineral oil for $10 \mathrm{~min}$ at ZT4 under light and at ZT22 under red light $(n=6$ rats per ZT). We exposed rats to odorant in a separate room to avoid stimulation of neighbouring rats. A filter paper, soaked with $200 \mu \mathrm{L}$ of the odorant, was placed on top of cage in a metal teaball. Rats were killed $50 \mathrm{~min}$ after odorant removal, olfactory bulbs were collected and post-fixed overnight at $4{ }^{\circ} \mathrm{C}$ in $4 \%$ paraformaldehyde PBS. Tissues were cryo-protected with sucrose (30\%) and cryo-sectioned coronally (20 $\mu \mathrm{m}$ thick). Sections were kept frozen at $-80{ }^{\circ} \mathrm{C}$ until use. Nonspecific staining was blocked by incubation for $1 \mathrm{~h}$ at room temperature with nonimmune goat serum at a dilution $1: 10$ in phosphate-buffered saline (PBS) containing $0.5 \%$ Triton $\mathrm{X}-100$ and $2 \%$ bovine serum albumin (PBS/TX/BSA). The sections were then incubated for $48 \mathrm{~h}$ at $4{ }^{\circ} \mathrm{C}$ with a rabbit polyclonal antibody to cFos protein (Ab-5, 1 : 2000; Calbiochem, San Diego, CA, USA) in PBS/TX/ BSA. After washes in PBS, labelling was visualised using secondary antibody conjugated with biotin $(1: 200,2 \mathrm{~h}$ at room temperature) revealed by an avidin-peroxidase kit (ABC standard kit; Vector, Burlingame, CA, USA) with diaminobenzidine (SigmaAldrich) (DAB $0.05 \%$ ) and $\mathrm{H}_{2} \mathrm{O}_{2}(0.0015 \%)$. Finally, slides were rinsed in PBS, mounted in Depex (Fisher Scientific) and acquisitions were performed using a DP50 CCD camera (Olympus). For each animal, we took six images located dorsally, medially and ventrally. Images at $\times 20$ magnification were selected by two observers blind to the treatments and analysed using IMAGEJ software to threshold specific cFos staining as described previously (Laziz et al., 2011). The same parameters were applied for all images and cFos staining was assessed in the glomerular layer, the mitral layer and the granular layer from all cells of the visual field.
Results were expressed as a relative value compared to ZT22 group.

\section{Statistical analysis}

Data are expressed as mean \pm standard error of the mean (SEM). Student's $t$ test was used to determine the statistical differences between groups except for EOG dose-response curves, which were statistically analysed with a two-way ANOvA, and for qPCR and liver/body weight ratio results, which were analysed using a oneway ANOVA followed by Bonferroni multiple comparison post hoc test. A probability value of $P<0.05$ was used as an indication of significant differences.

\section{Results \\ Olfactory epithelium responses to odorants vary during the day}

To investigate the daily variations of the $\mathrm{OE}$ sensitivity to odorant stimulations, we performed EOG recordings at four zeitgeber (ZT) times. We chose those four ZT times (ZT4, ZT10, ZT16 and ZT22) to record a group of six rats kept in the same illumination condition for each ZT time (Fig. 1A). We stimulated the OE with increasing concentrations of limonene and hexanal and measured the maximum amplitude of responses to odorant (Fig. 1B). The shape of the dose response to these odours was similar to those found in previous work (Raynaud et al., 2015). Two-way ANOva analysis revealed statistical differences between the curves due to time for both limonene $\left(F_{3,126}=5.94 ; \quad P=0.0008\right) \quad$ and hexanal $\quad\left(F_{3,208}=6.82\right.$; $P=0.0002)$. Average recorded traces for two concentrations of limonene (Fig. 1C) and hexanal (Fig. 1D) highlight the maximum amplitude difference between ZT4 and ZT22. The amplitude of EOG recordings following stimulation without odorant was always around $1 \mathrm{mV}$. This amplitude reflects mainly mechanical detection of the airflow change (Chen et al., 2012). To exclude that the daily oscillation of EOG signals were linked to this mechanical response, we examined the relative response to ZT22 between the stimulation with the lowest concentrations of limonene and hexanal where the mechanical response would have the maximum effect. At those concentrations, limonene and hexanal give very similar responses (Fig. 1B), thus the daily variation in this ratio should be similar for both odours if our observed effect was solely due to a daily oscillation of this mechanical response. It was not as the variation in responses to these low concentrations was significantly different between hexanal and limonene (Fig. $1 \mathrm{E}, F_{1,84}=6.71 ; P=0.011$ ). Furthermore, the OE sensitivity to odorant stimulations was the highest at ZT4 and then decreased to attain its lowest value at ZT22. On the basis of this result, we compared the OE normalised responses between ZT4 and ZT22 for all the concentrations of odorants (Fig. 1F, G). The ZT variations observed were significantly different for the lowest concentrations of limonene $\left(F_{1,44}=9.01\right.$, $P=0.0066)$ and hexanal $\left(F_{1,88}=13.65, P=0.0013\right)$.

\section{OSN signal transduction genes and proteins fluctuations are associated with $O E$ sensitivity variations}

To investigate the molecular events associated with the variations of $\mathrm{OE}$ sensitivity to odorant stimulations during the day, we quantified the expression level of genes related to the olfactory transduction pathway. During EOG experiments, one hemi-head was used for recordings. We collected the OE from the second hemi- 
A

4 ZT points

6 rats per point $/ 30$ min recording each (1 hemi-head for EOG, 1 hemi-head for qPCR)

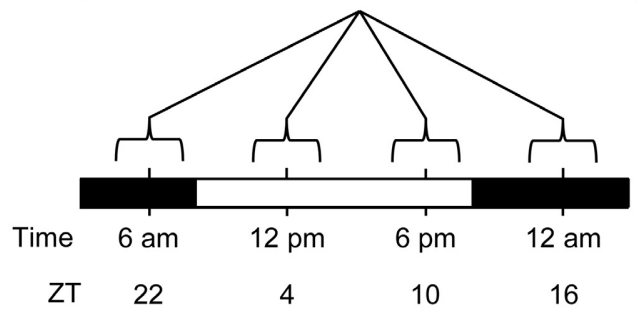

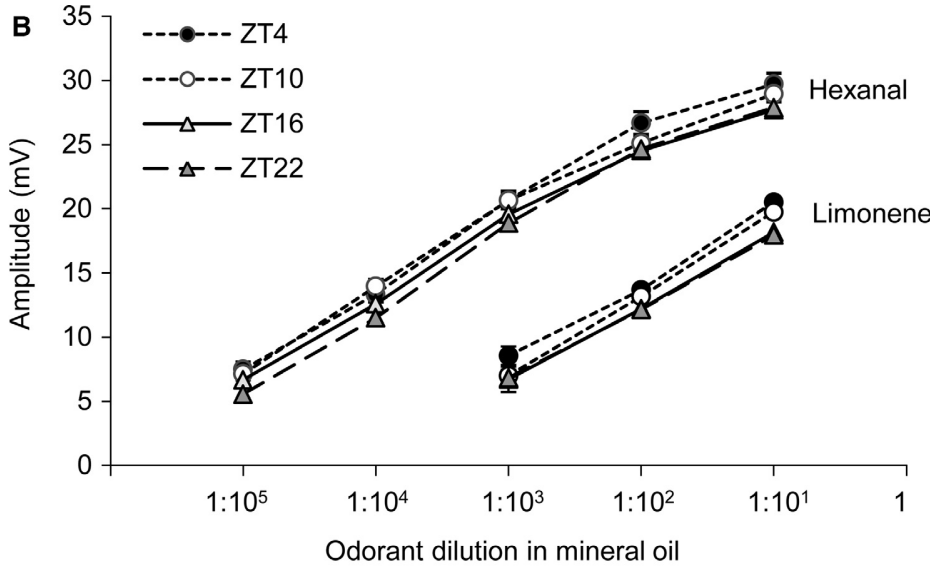

\section{C \\ Limonene 1:10 \\ in mineral oil v/v}
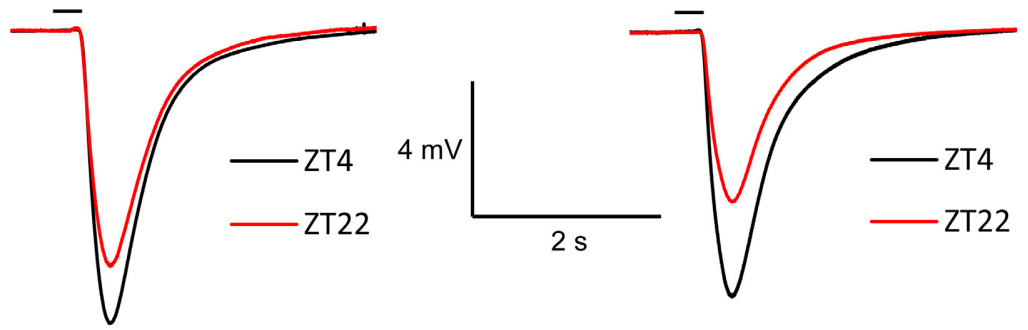

Hexanal $1: 10^{5}$
E

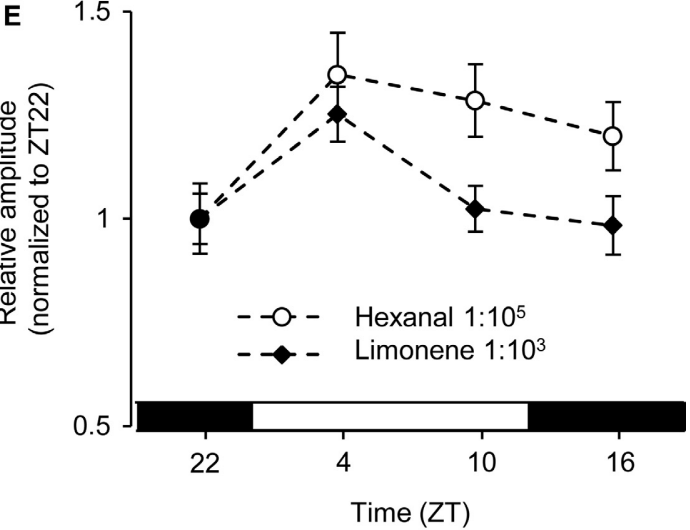

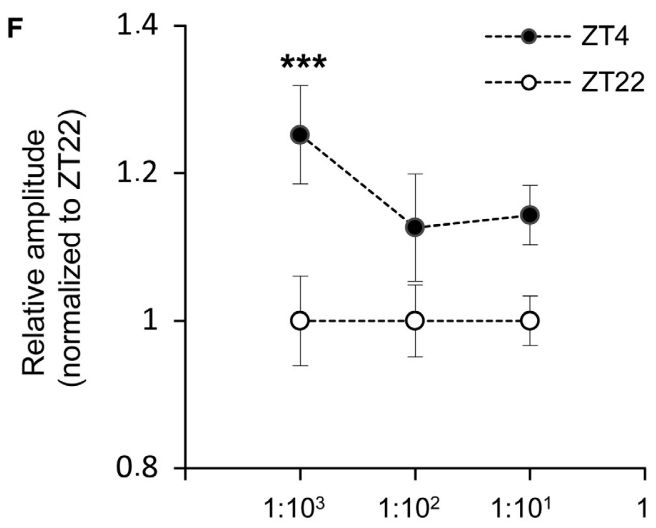

Limonene dilution in mineral oil

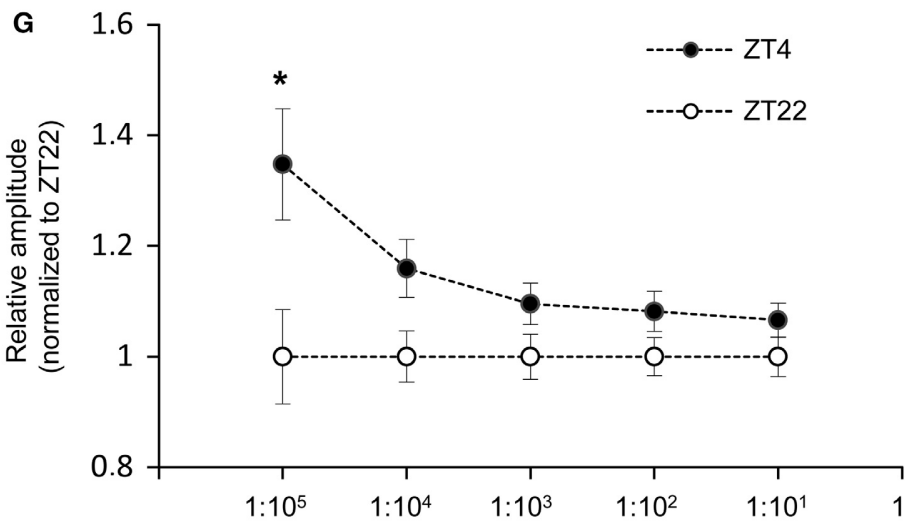

Hexanal dilution in mineral oil

FIG. 1. Daily variations in the response of the olfactory epithelium to odorants. (A) Recording protocol. (B) EOG dose-response for limonene and hexanal depending on ZT. Values represent the mean of peak amplitudes ( \pm SEM) values. (C) Average EOG traces of responses to $200 \mathrm{~ms}$ of limonene or (D) hexanal stimulus at ZT4 and ZT22. (E) EOG signal amplitude relative to ZT22 for the lowest concentrations of hexanal and limonene. Values represent the mean of the relative peak amplitudes $( \pm$ SEM). (F) Relative amplitude at ZT4 for limonene and $(\mathrm{G})$ hexanal normalised to the corresponding ZT22 amplitude (two-way repeated measure ANOva followed by Bonferroni post test; $\left.* * * P<0.001,{ }^{*} P<0.05\right)$. Values represent the mean of relative peak amplitudes $( \pm$ SEM). $n=6$ replicates. [Colour figure can be viewed at wileyonlinelibrary.com].

head to perform the qPCR on the same animals. We measured the expression of the only two deorphaned olfactory receptors (OR) in rats, i7/Olr 226 and U131/Olr 1278 (Murrell \& Hunter, 1999; Glatz \& Bailey, 2011; Khan et al., 2011). Their transcript levels varied throughout the day and were higher at ZT22 (statistically significant for U131) (Fig. 2A). We also evaluated the mRNA levels of olfactory-type $G$ protein $\left(\mathrm{G}_{\text {olf }}\right)$, adenylyl cyclase III (ACIII), cyclic nucleotide gated channel alpha 2 (Cnga2) and phosphodiesterase PDE1C2, four genes encoding proteins implicated in the odorant induced potential receptor in OSN (Kleene, 2008). $\mathrm{G}_{\text {olf }}$, ACIII and PDE1C2 mRNA levels exhibited patterns similar to that of ORs, with a maximum at ZT22 (Fig. 2B). It was also similar with $\mathrm{Cng} 2$ but this did not reach significance. Next, we examined by western blot whether the protein level of $\mathrm{G}_{\text {olf }}$ and ACIII were also modified. As this required new animals, we focused only on ZT4 and ZT22, where EOG amplitude and level of mRNA related to transduction pathway were the most different. Both $\mathrm{G}_{\text {olf }}$ and ACIII were more abundant at ZT4 (Fig. 2C), which 


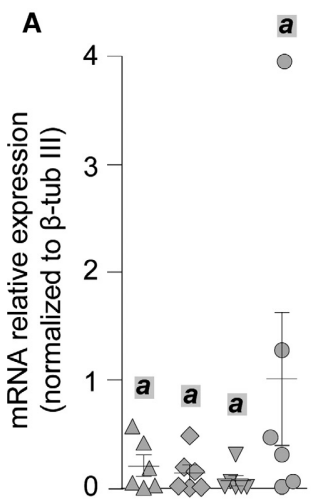

17

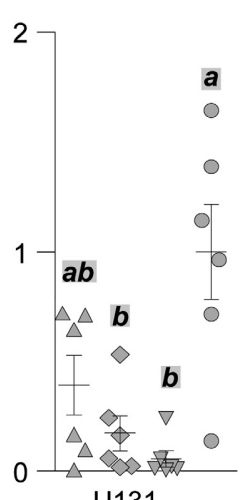

U131
B

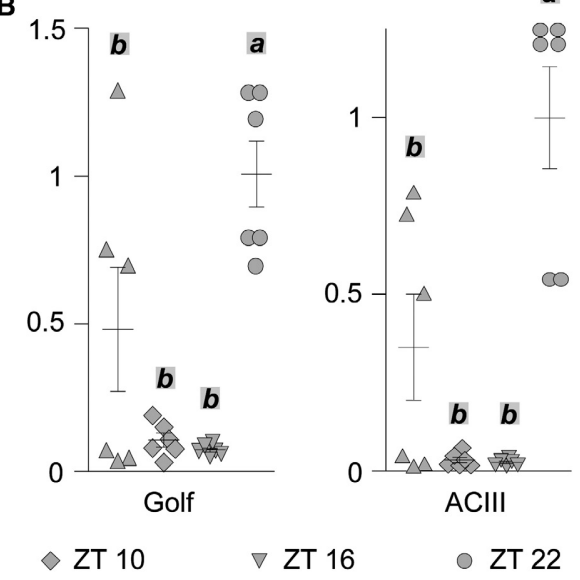

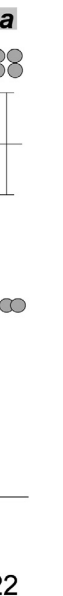
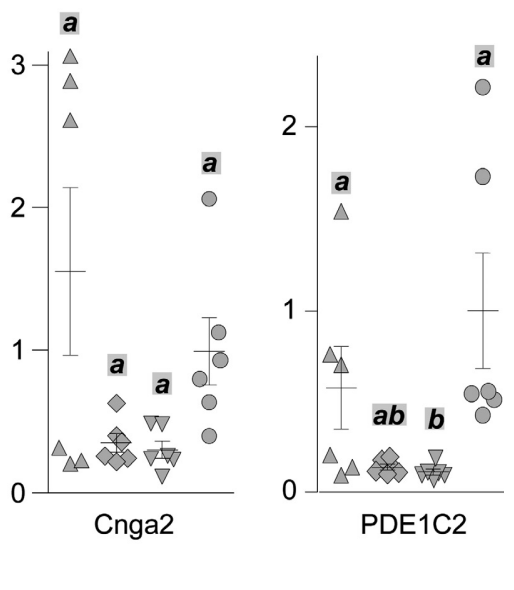

$\triangle \mathrm{ZT} 4$
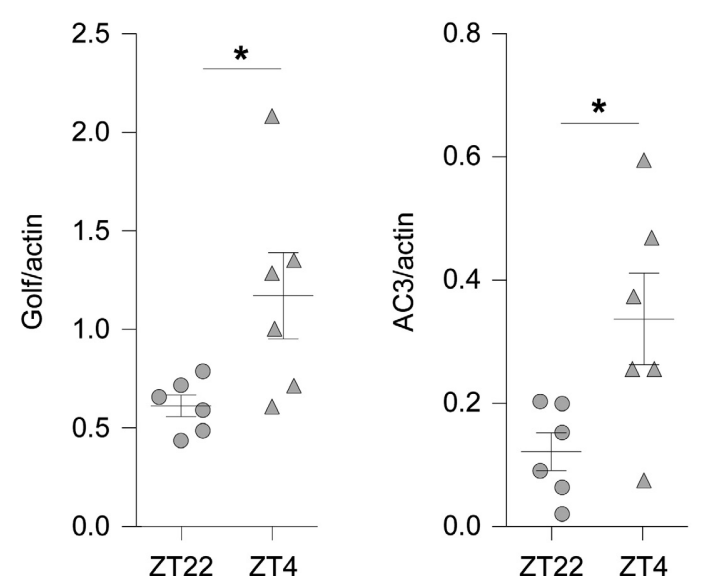

FIG. 2. ORs and signal transduction actors are expressed differentially during the day. qPCR analysis of (A) i7 and U131 ORs expression and (B) $\mathrm{G}_{\text {olf }}$, ACIII, Cnga2 and PDE1C2 in the olfactory mucosa depending on ZT. All genes are normalised to ZT22. Scatter plots represent means \pm SD ( $n=6$ replicates). Scatter plots with same letters are not significantly different (1-way ANovA; Newman-Keuls Multiple Comparison Test; $P<0.05)(\mathrm{C})$ Western blot analysis of $\mathrm{G}_{\text {olf }}$ and ACIII expression in the olfactory mucosa at ZT22 and ZT4. $(n=6$ replicates; Student's $t$ test; $* P<0.05)$.

was consistent with the time of the highest EOG amplitudes (Fig. 1E).

\section{OSNs transduction capacities vary during the day but not excitability or spontaneous activity}

To monitor whether OSNs exhibit daily variations in their physiological properties, as suggested by EOG, qPCR and western blot experiments, we performed patch-clamp recordings on rats' OSNs at ZT4 and ZT22. These recordings were carried out on the dendritic knobs of randomly chosen OSNs in an intact preparation as described earlier (Cadiou et al., 2014; Jarriault \& Grosmaitre, 2015). To characterise the total capacity of the transduction pathway, we stimulated OSNs at ZT4 $(n=19$ neurons) and ZT22 $(n=21$ neurons $)$ with a mix of $100 \mu \mathrm{M}$ IBMX and $10 \mu \mathrm{M}$ Forskolin. In voltage-clamp mode, rats' OSNs responded to IBMX + Forskolin with inward currents (Fig. 3A $\mathrm{A}_{\mathrm{a}}$ ). OSNs recorded at ZT22 exhibited a lower maximum amplitude and a smaller total current (Fig. 3A). Meanwhile, there was no difference between ZT4 and ZT22 for the kinetic parameters (data not shown). The same OSNs did not respond differently to stimulation with Ringer alone when recorded at ZT4 or ZT22 for any parameters analysed (maximum amplitude on Fig. 3A, other parameters not shown). The excitability of the OSNs was measured under current clamp by the injection of a $7 \mathrm{pA}$ depolarising current (Fig. 3B $\mathrm{B}_{\mathrm{a}}$ ). The characteristics of the responses to the depolarising currents did not differ between OSNs recorded at ZT4 $(n=37)$ or ZT22 $(n=44)$ : no difference was observed for the average firing frequency (Fig. $3 \mathrm{~B}_{\mathrm{b}}$ ), the latency (Fig. $3 \mathrm{~B}_{\mathrm{c}}$ ) and the action potential train duration (Fig. $3 \mathrm{~B}_{\mathrm{d}}$ ). We also recorded the spontaneous firing activity of the same OSNs in the current-clamp configuration and did not observe any difference (Fig. 3C). In conclusion, OSNs exhibited larger responses to the IBMX + Forskolin mix when recorded at ZT4, confirming the ZT/ time-dependent variations in olfactory transduction pathway while their excitability was similar.

\section{$O B$ cFos activation coincides with OE activity peak}

Using animals maintained in $12 \mathrm{~L}: 12 \mathrm{D}$ condition, we observed that $\mathrm{OE}$ responses to odorant stimulation peaked during the light phase. However, in previous studies using animals in constant darkness condition, OB activity has been shown to be higher during the dark phase (Granados-Fuentes et al., 2006). As it would be surprising that $\mathrm{OE}$ and $\mathrm{OB}$ follow different daily oscillation of olfactory sensitivity, we evaluated daily variations of activity in the $\mathrm{OB}$ with our experimental conditions. We measured odour induced $c F$ os expression in glomerular, mitral cell and granular cell layers of the $\mathrm{OB}$ in a new cohort of animals (Fig. 4A). Labelling revealed a higher 
$\mathrm{Aa}$

IBMX + Forskolin

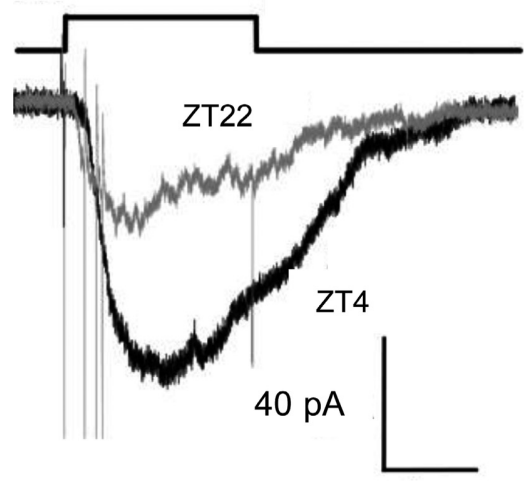

$1 \mathrm{~s}$
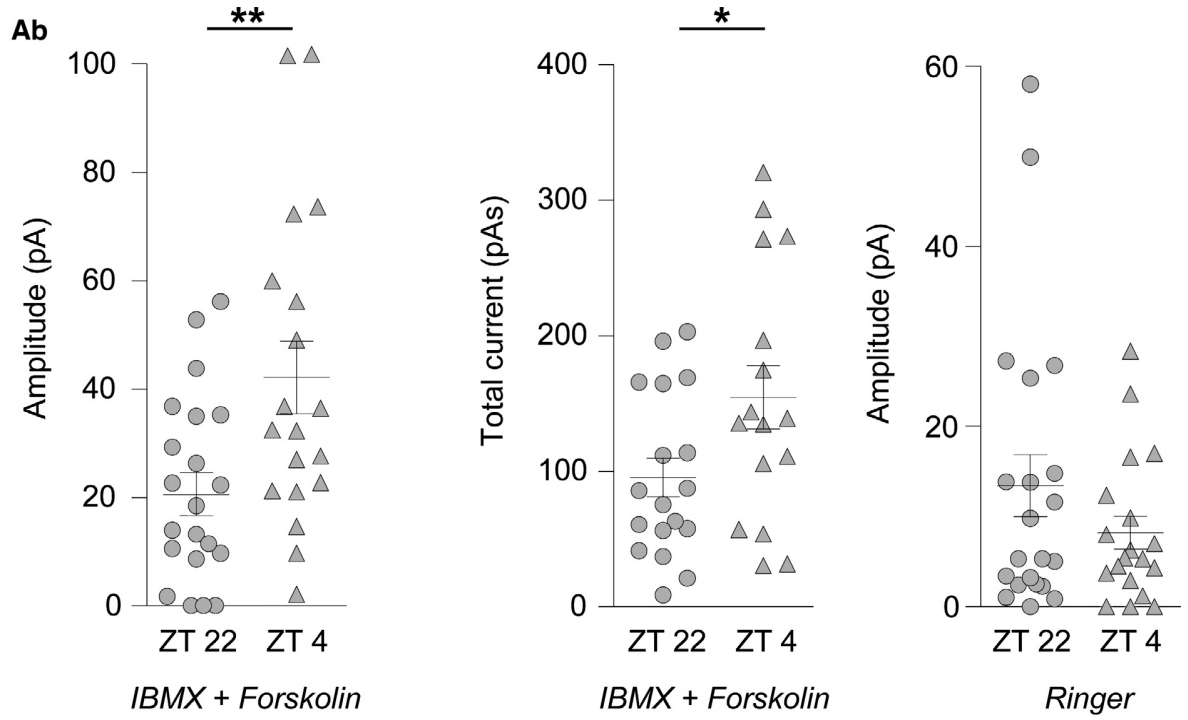

$\mathrm{Ba}$
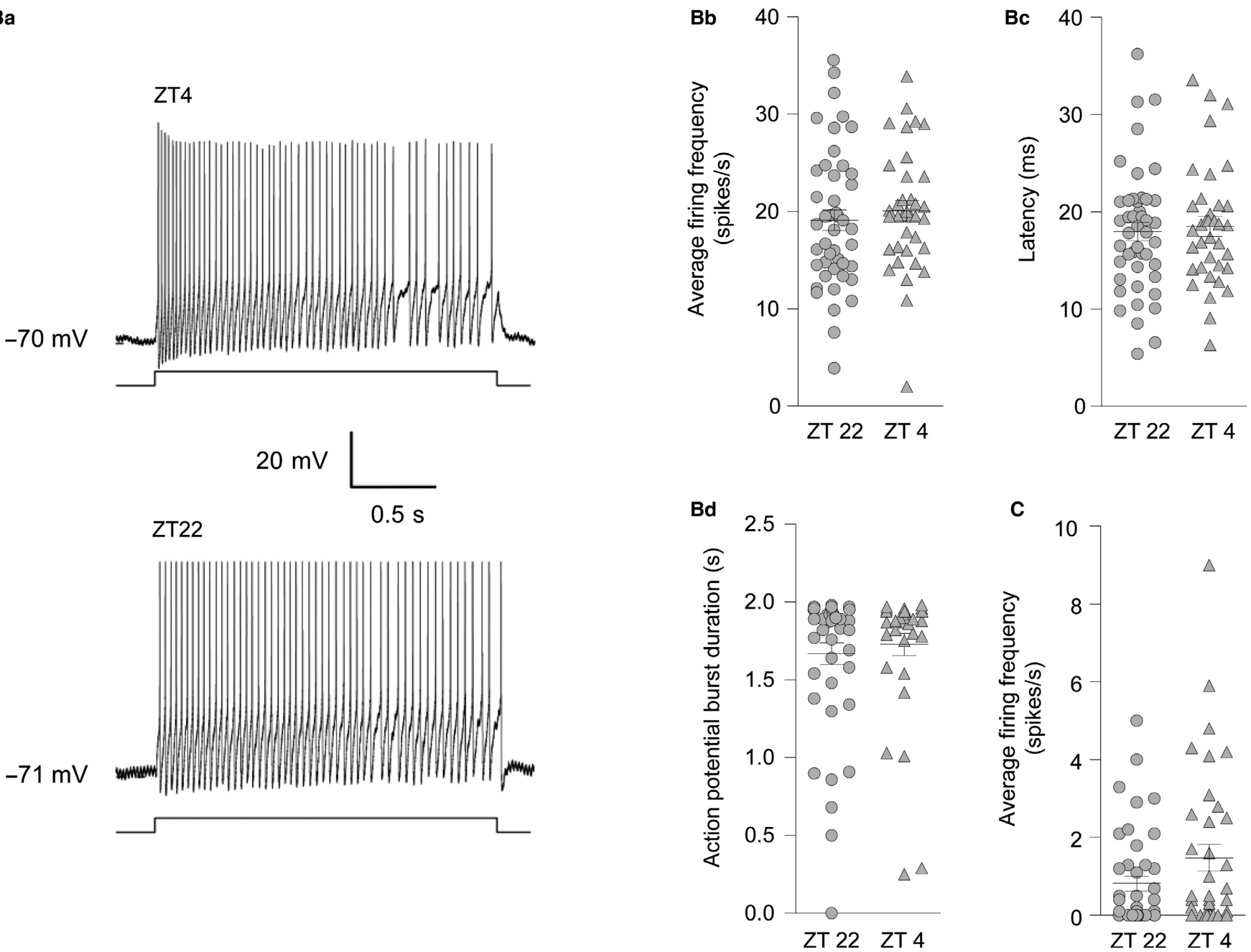

FIG. 3. Daily variations in individual olfactory sensory neurons properties. ( $\mathrm{A}_{a}$ ) Representative responses of OSNs to a $2 \mathrm{~s}$ stimulation with $100 \mu \mathrm{M}$ IBMX + $10 \mu \mathrm{M}$ Forskolin mix. $\left(\mathrm{A}_{\mathrm{b}}\right)$ Analysis of the maximum amplitude and the total current at ZT4 $(n=19)$ and ZT22 $(n=21)$. ( $\left.\mathrm{B}_{\mathrm{a}}\right)$ Representative examples of action potentials elicited by a 7 pA excitatory current. $\left(\mathrm{B}_{\mathrm{b}-\mathrm{d}}\right)$ Quantification of the excitability in ZT4 $(n=37)$ and ZT22 $(n=44)$ of recorded OSNs: scatter plots representing the average firing frequency of action potentials elicited by $7 \mathrm{pA}$ current $\left(\mathrm{B}_{\mathrm{b}}\right)$, the latency between the onset of the stimulus and the first spike $\left(\mathrm{B}_{\mathrm{c}}\right)$ and the average duration of the spike train elicited $\left(\mathrm{B}_{\mathrm{d}}\right)$. (C) Average spontaneous firing frequency of ZT4 $(n=37)$ and ZT22 ( $\left.n=44\right)$ recorded OSNs. All recordings performed in perforated patch and at a membrane potential of $-70 \mathrm{mV}$ (Student's $t$ test; $* P<0.05 ; * * P<0.01$ ). 


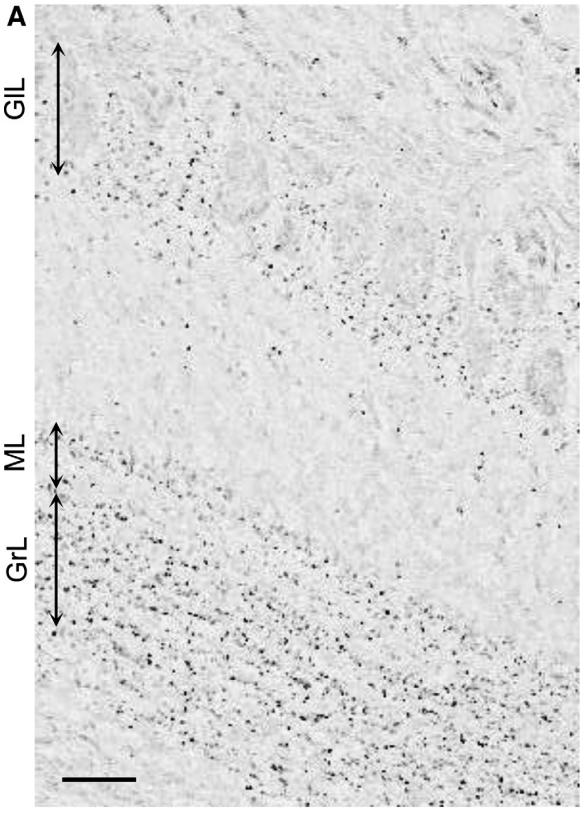

ZT22

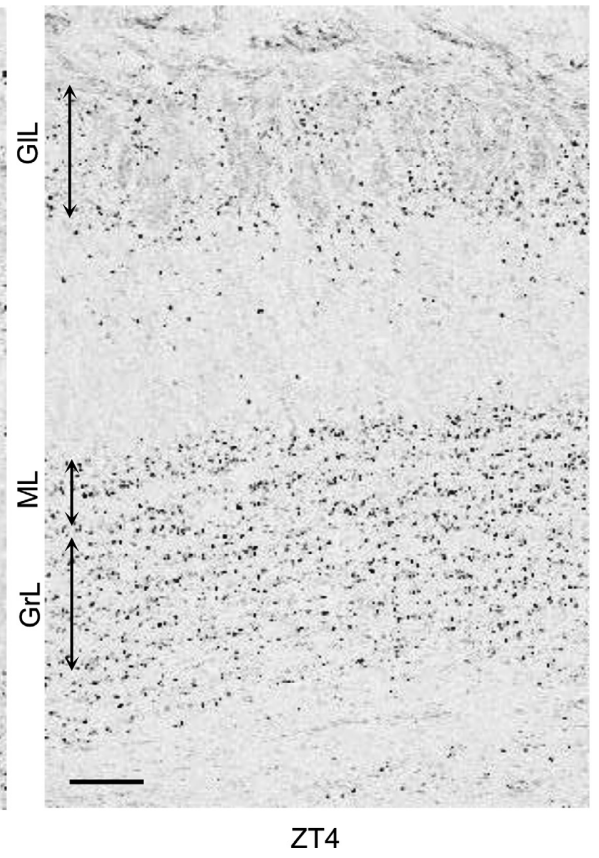

B

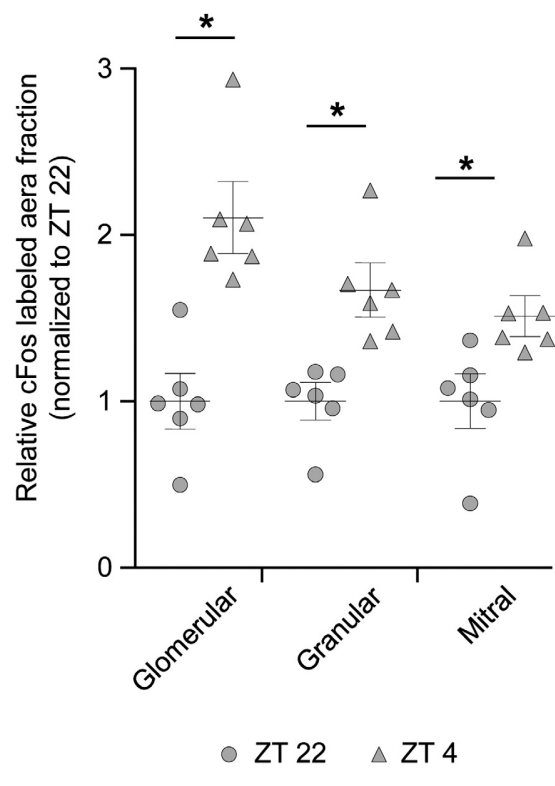

FIG. 4. Variations of odour-evoked cFos expression in the OB during the day. (A) Two representative pictures of cFos labelling for this experiment. Scale bar $=100 \mu \mathrm{m}$. (B) cFos labelled area normalised to ZT22 for the glomerular layer (GIL), the mitral cell layer (ML) and the granular cell layer (GrL) at ZT4 and ZT22. ( $n=6$ replicates; Student's $t$ test; $* P<0.05)$.

expression at ZT4 than ZT22 in the three layers (Fig. 4B). Thus, in our experimental protocol the variation in $\mathrm{OB}$ activity were concomitant to those in the $\mathrm{OE}$.

\section{OE sensitivity rhythm is not impaired by food deprivation}

The metabolic status is well known to fluctuate in relation to sleepwake and food intake rhythms (Dibner et al., 2010). Since it has been established that the metabolic status of an organism influences its olfaction (Lucero, 2013), we wanted to investigate a possible link between this metabolism and daily variations in OE sensitivity. We carried out a new experiment with 16-h food-deprived rats.

In parallel to EOG recordings, we measured the liver weight to simply evaluate the consequences of the fasting on the metabolic status of the recorded animals. In the first experiment, liver weight fluctuated in fed rats over the sampling period, whereas this was abolished in food-deprived animals (Fig. 5A). This result indicates that $16 \mathrm{~h}$ food deprivation modified rats' metabolic status: this food deprivation paradigm levels the metabolic status between the four groups. Using the same experimental protocol as before (Fig. 1A), we stimulated $\mathrm{OE}$ with increasing concentrations of limonene and hexanal and measured maximum amplitude of responses to different odorants (Fig. 5B). Statistical analysis showed that curves were different depending on the $\mathrm{ZT}$ for both limonene $\left(\mathrm{F}_{(3,132)}=13.1\right.$; $P<0.0001)$ and hexanal $\left(\mathrm{F}_{(3,220)}=21.07 ; P<0.0001\right)$. Daily variations of $\mathrm{OE}$ sensitivity were still apparent although maximum EOG responses were recorded at ZT10 for the lowest limonene concentration and at ZT4 for the lowest hexanal concentration (Fig. 5C). In a similar fashion to the fed animals (Fig. 1), the relative ZT4 and ZT22 maximum amplitude response were significantly different for the lowest concentrations of limonene and for the two lowest concentrations of hexanal $\left(\mathrm{F}_{1,44}=11.53, P=0.0026\right.$ and $\mathrm{F}_{1,88}=12.80$, $P=0.0017$ respectively; Fig. 5D and E). The lowest concentration of odorant for hexanal was the most different as the depolarisation amplitude values are respectively 4.34 vs. $6.03 \mathrm{mV}$ for the lowest concentration of hexanal (a change of $43 \%$ ) while they are 24.07 vs. $28.63 \mathrm{mV}$ for the highest concentration (a change of 19\%). Taken together, these results show that the daily variations of OE sensitivity are not driven by the metabolic status of the animal and that food deprivation even enhances the amplitude of these daily fluctuations as significant differences were observed for a wider range of odorant dilution in starved animals.

\section{Discussion}

Daily oscillation of neuronal activity in the OB has been described in numerous studies (Dibner et al., 2010). OB inputs from the OE are known to be modulated by several factors, such as the metabolic status of the animals (Lacroix et al., 2008) or stress (Raynaud et al., 2015). Consequently, the efficiency of peripheral odorant detection could also oscillate daily. In this study, we investigated nycthemeral variations of the functional response of the OE. EOG signals and patch-clamp recordings of OSN show an oscillation of $20 \%$ in their amplitude. Such level of variation has been reported earlier when studying the effect of metabolic hormones (Lacroix et al., 2008; Negroni et al., 2012), indicating that the nycthemeral oscillations observed here are within the physiological range of regulatory processes. These differences were mainly significant for the detection of low concentrations of odorants which makes sense from a physiological point of view: low concentrations of odorants correspond to levels that animals encounter in their environment.

Real-time PCR analyses revealed that OR and signal transduction gene expression peak $6 \mathrm{~h}$ prior to maximum EOG responses (Fig. 2). Considering that there should be a delay between transcription and translation peak, we made the assumption that mRNA upregulation would lead to higher protein concentration a few hours later. Western blot results show that ACIII and $\mathrm{G}_{\text {olf }}$ proteins are indeed more abundant in the $\mathrm{OE}$ concomitantly to $\mathrm{OE}$ responsiveness peak. EOG response reflects the receptor potential induced by the odorant arrival (Scott \& Scott-Johnson, 2002). As ACIII and 

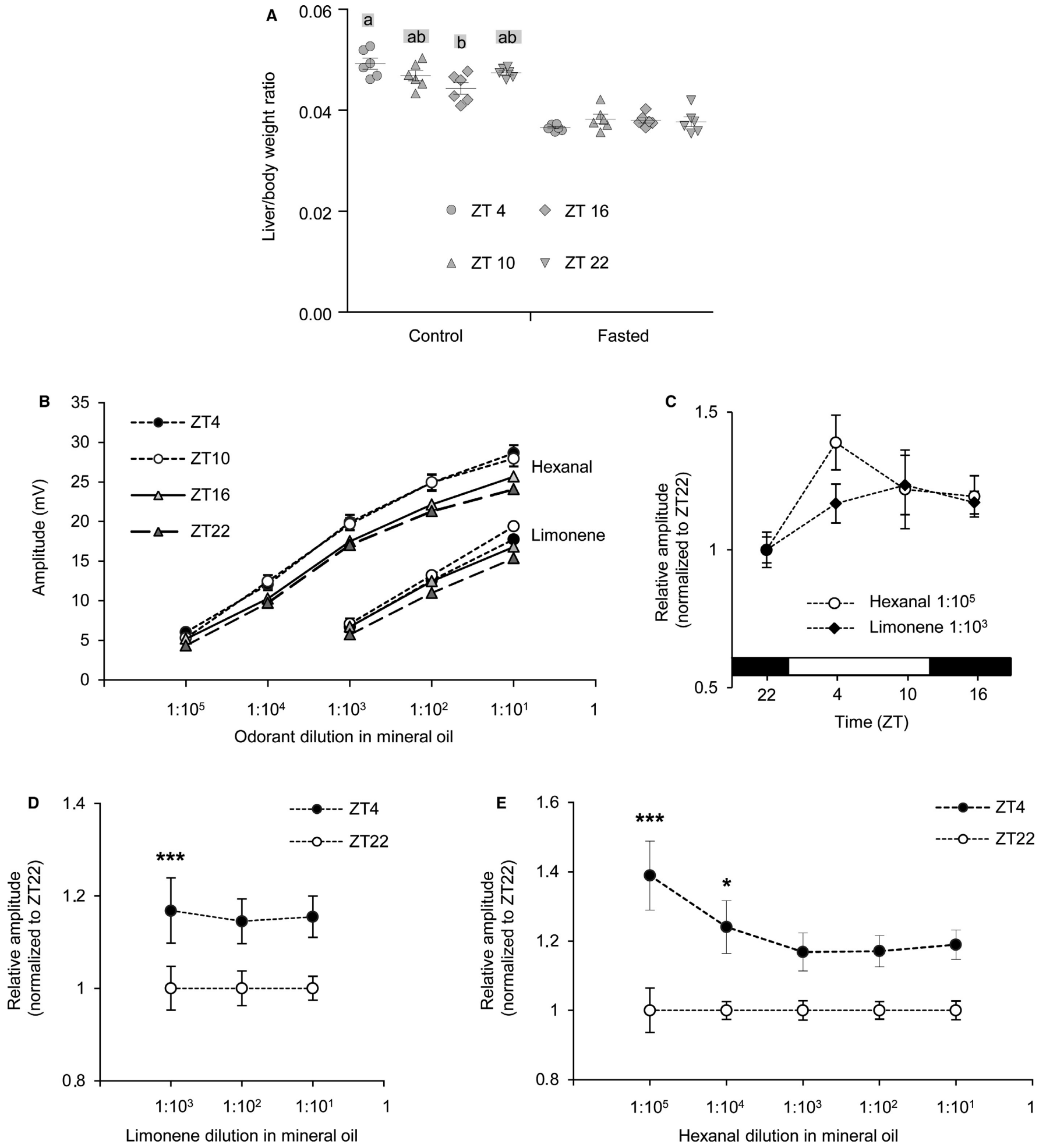

FIG. 5. Sixteen hours food deprivation does not abolish daily rhythm of olfactory epithelium functional responses. (A) Liver weight normalised to body weight during the day with free access to food or after $16 \mathrm{~h}$ food deprivation (ZT time indicated corresponding to the end of the $16 \mathrm{~h}$ food restriction). Scatter plots with same letters are not significantly different (one-way ANova; Newman-Keuls Multiple Comparison Test; $P<0.05$ ) (B) Mean signal amplitude for hexanal and limonene varies according to ZT. Values represent the mean of peak amplitudes ( \pm SEM) values. (C) EOG signal amplitude for hexanal and limonene relative to ZT22. (D) Relative amplitude at ZT4 for limonene and (E) hexanal normalised to corresponding ZT22 amplitude (two-way repeated measure ANova followed by Bonferroni post test; $* P<0.05 ; * * * P<0.001) . n=6$ replicates.

$\mathrm{G}_{\mathrm{olf}}$ are key actors of the transduction pathway, an increase in their amount will improve the transduction pathway efficiency and raise the probability of cyclic nucleotide and calcium dependant channel opening. This higher level of transduction proteins should thus affect OSN physiological properties recorded in patch-clamp. Indeed, those recordings show that the amplitude of OSN responses 
was also higher at ZT4 when ACIII and $\mathrm{G}_{\text {olf }}$ proteins were more abundant.

The increase in ACIII and $\mathrm{G}_{\text {olf }}$ proteins level could potentially have an impact on basal firing rate of OSN action potentials as ACIII has a constitutive basal level of activity. However, we did not observe any difference (Fig. 3C). This may be due to the already very low basal firing activity recorded in OSNs at ZT4. Another hypothesis may be that by recording randomly chosen OSNs expressing diverse ORs, a greater distribution of spontaneous firing frequencies was recorded hiding potential variation in individual populations (Connelly et al., 2013).

Olfactory sensory neurons are also known to be sensitive to mechanical stimulation through ACIII (Chen et al., 2012). As ACIII level is increased at ZT4 compared to ZT22, one could expect to have an increase in the OSN level of stimulation with Ringer alone at ZT4 compared to ZT22 during patch-clamp recordings. We did not observe any significant difference (Fig. 3C). It cannot be excluded that the mechanical sensitive pathway in OSNs requires other components than ACIII and those components do not oscillate daily in their gene expression level. Furthermore, the mechanical difference may be too small to be statistically significant. Overall, these results clearly show that the nycthemeral variations of $\mathrm{OE}$ responsiveness are correlated with gene regulation.

We found in both EOG and patch-clamp recordings that the OE responsiveness peaks during the light phase, which is the resting phase of rodents. As sleep in rodents is heavily fragmented with an average of 100 waking episodes per day (Van Twyver, 1969), an increased sensitivity during the rest phase would improve the olfactory vigilance during the waking episodes of the resting phase. It would allow rats to be more reactive, for example, to predator odours. However, this result is in contradiction with previous observation of daily variation in olfactory sensitivity performed with animal in DD condition. Indeed, when maintained in constant darkness, mice display improved odour detection performances in their active phase (Granados-Fuentes et al., 2011; Miller et al., 2014). Similarly, while we observed in rat a daily variation in the expression of genes related to the olfactory transduction pathway in the OE, their expression did not change in mice maintained in constant darkness (Saleh et al., 2015); although genes such as Kirrel2 related to OSN activity did. Most previous studies showed as well an increased OB activity over the dark phase in rodents (Amir et al., 1999; Funk \& Amir, 2000; Granados-Fuentes et al., 2006). We thus wanted to evaluate the correlation between $\mathrm{OB}$ activity variations and $\mathrm{OE}$ responsiveness with our model. We measured $c$ Fos expression as a marker of cellular activity (Onoda, 1992) in rats OB after exposure to a complex odour (almond oil) at ZT4 and ZT22. Results point out an increase in $c F$ os area in the OB of animals taken at ZT4 compared to ZT22, in agreement with our observed variation in the OE. Between our study and others, the main differences are that we used rats maintained on light-dark cycle. The observed discrepancy may thus be related to the difference of species and lighting condition. Finally, the daily variation in olfactory sensitivity was different among odorant (Fig. 1E) as was the time of the maximum of sensitivity for limonene between fed (ZT 4) and starved animals (ZT10). It is thus difficult to generalise the time of maximum olfactory sensitivity that may vary according to odorant identity or animal metabolic status. Still, our work is consistent with previous studies as it demonstrates that the olfactory sensitivity varies daily and point out for the first time that this variation occurs as early as the receptor potential in the OSNs.

It is well established that sleep-wake and feeding-fasting cycles induce metabolic variations which follow a circadian pattern
(Bailey et al., 2014). For this reason, we investigated a possible link between nycthemeral variations of OE responsiveness and the metabolic status. Using a food deprivation approach, we partially abolished the metabolic daily fluctuations of our rats as shown with the stability of liver weight in this cohort (Fig. 5A). Despite a stable daily metabolic status, the EOG signals from fasted animals followed a similar pattern over the course of the day compared to animals fed ad libitum. Thus, daily variations in $\mathrm{OE}$ sensitivity are not solely driven by the metabolic status.

Given its close functional and structural proximity to the $\mathrm{OB}$, the question of the relationship between the $\mathrm{OE}$ and $\mathrm{OB}$ oscillators is challenging to unravel experimentally and remains to be clarified. Three hypotheses could be formed: (i) $\mathrm{OE}$ responsiveness variations are independent from the $\mathrm{OB}$, (ii) the $\mathrm{OB}$, which can be entrained by the SCN, drives functional changes in the $\mathrm{OE}$ throughout the day or (iii) $\mathrm{OE}$ sensitivity fluctuations may lead to a rhythm in $\mathrm{OB}$ activity. Interestingly, periodicity in environmental odorant levels has been recently identified as a potential zeitgeber (Abraham et al., 2013). This could be exploited to test some of the above hypotheses.

In conclusion, for the first time in rat, we provide electrophysiological and molecular evidence that the OE sensitivity varies during the day. This phenomenon appears independent from metabolic influence and, as the $\mathrm{OE}$ functions in close proximity to the $\mathrm{OB}$, their relationship around the clock requires additional characterisation.

\section{Acknowledgement}

This work was funded by the Institut National de la Recherche Agronomique (INRA). We would like to thank Birte Nielsen for her help improving the manuscript, all lab members for fruitful discussions, Patrice Dahirel and Aurélien Raynaud for animal care and treatments and Didier Durieux for technical help. XG and DJ would like to thank the CSGA animal facility for animal care.

\section{Conflict of interests}

The authors declare no conflict of interests.

\section{Author contributions}

$\mathrm{AF}$ and NM designed and performed experiments, analysed data and wrote the paper; VB and DJ designed and performed experiments and analysed data; XG designed experiments and analysed data; DG, AA performed experiments; VB, XG and DJ edited the manuscript.

\section{Data accessibility}

The raw data can be uploaded from the website figshare.com. https://doi.org/10.6084/m9.figshare.4888568.v1.

\section{Abbreviations}

EOG, Electroolfactogram; OB, Olfactory Bulb; OE, Olfactory Epithelium; OSN, Olfactory Sensory Neurons; SCN, Suprachiasmatic nucleus; ZT, Zeitgeber times.

\section{References}

Abraham, U., Saleh, M. \& Kramer, A. (2013) Odor is a time cue for circadian behavior. J. Biol. Rhythms, 28, 26-37. 
Amir, S., Cain, S., Sullivan, J., Robinson, B. \& Stewart, J. (1999) In rats, odor-induced Fos in the olfactory pathways depends on the phase of the circadian clock. Neurosci. Lett., 272, 175-178.

Bailey, S.M., Udoh, U.S. \& Young, M.E. (2014) Circadian regulation of metabolism. J. Endocrinol., 222, R75-R96.

Cadiou, H., Aoude, I., Tazir, B., Molinas, A., Fenech, C., Meunier, N. \& Grosmaitre, X. (2014) Postnatal odorant exposure induces peripheral olfactory plasticity at the cellular level. J. Neurosci., 34, 4857-4870.

Chen, X., Xia, Z. \& Storm, D.R. (2012) Stimulation of electro-olfactogram responses in the main olfactory epithelia by airflow depends on the type 3 adenylyl cyclase. J. Neurosci., 32, 15769-15778.

Connelly, T., Savigner, A. \& Ma, M. (2013) Spontaneous and sensoryevoked activity in mouse olfactory sensory neurons with defined odoran receptors. J. Neurophysiol., 110, 55-62.

Dibner, C., Schibler, U. \& Albrecht, U. (2010) The mammalian circadian timing system: organization and coordination of central and peripheral clocks. Annu. Rev. Physiol., 72, 517-549.

Firestein, S., Darrow, B. \& Shepherd, G.M. (1991) Activation of the sensory current in salamander olfactory receptor neurons depends on a $\mathrm{G}$ proteinmediated cAMP second messenger system. Neuron, 6, 825-835.

Francois, A., Laziz, I., Rimbaud, S., Grebert, D., Durieux, D., Pajot-Augy, E. \& Meunier, N. (2013) Early survival factor deprivation in the olfactory epithelium enhances activity-driven survival. Front Cell Neurosci., 7, 271

Funk, D. \& Amir, S. (2000) Circadian modulation of fos responses to odor of the red fox, a rodent predator, in the rat olfactory system. Brain Res. 866, 262-267.

Glatz, R. \& Bailey-Hill, K. (2011) Mimicking nature's noses: from receptor deorphaning to olfactory biosensing. Prog Neurobio, 93, 270-296.

Granados-Fuentes, D., Saxena, M.T., Prolo, L.M., Aton, S.J. \& Herzog, E.D. (2004) Olfactory bulb neurons express functional, entrainable circadian rhythms. Eur. J. Neurosci., 19, 898-906.

Granados-Fuentes, D., Tseng, A. \& Herzog, E.D. (2006) A circadian clock in the olfactory bulb controls olfactory responsivity. J. Neurosci., 26, 12219-12225.

Granados-Fuentes, D., Ben-Josef, G., Perry, G., Wilson, D.A., Sullivan-Wilson, A. \& Herzog, E.D. (2011) Daily rhythms in olfactory discrimination depend on clock genes but not the suprachiasmatic nucleus. J. Biol. Rhythms, 26, 552-560.

Grosmaitre, X., Santarelli, L.C., Tan, J., Luo, M. \& Ma, M. (2007) Dual functions of mammalian olfactory sensory neurons as odor detectors and mechanical sensors. Nat. Neurosci., 10, 348-354.

Honma, A., Yamada, Y., Nakamaru, Y., Fukuda, S., Honma, K. \& Honma, S. (2015) Glucocorticoids Reset the Nasal Circadian Clock in Mice. Endocrinology, 156, 4302-4311.

Jarriault, D. \& Grosmaitre, X. (2015) Perforated patch-clamp recording of mouse olfactory sensory neurons in intact neuroepithelium: functional analysis of neurons expressing an identified odorant receptor. J. Vis. Exp., e52652.

Khan, M., Vaes, E. \& Mombaerts, P. (2011) Regulation of the probability of mouse odorant receptor gene choice. Cell, 147, 907-921.

Kleene, S.J. (2008) The electrochemical basis of odor transduction in vertebrate olfactory cilia. Chem. Senses, 33, 839-859.

Lacroix, M.C., Badonnel, K., Meunier, N., Tan, F., Schlegel-Le Poupon, C., Durieux, D., Monnerie, R., Baly, C. et al. (2008) Expression of insulin system in the olfactory epithelium: first approaches to its role and regulation. J. Neuroendocrinol., 20, 1176-1190.

Laziz, I., Larbi, A., Grebert, D., Sautel, M., Congar, P., Lacroix, M.C., Salesse, R. \& Meunier, N. (2011) Endothelin as a neuroprotective factor in the olfactory epithelium. Neuroscience, 172, 20-29.

Lowe, G. \& Gold, G.H. (1993) Nonlinear amplification by calcium-dependent chloride channels in olfactory receptor cells. Nature, 366, 283-286.

Lucero, M.T. (2013) Peripheral modulation of smell: fact or fiction? Semin. Cell Dev. Biol., 24, 58-70.

Miller, J.E., Granados-Fuentes, D., Wang, T., Marpegan, L., Holy, T.E. \& Herzog, E.D. (2014) Vasoactive intestinal polypeptide mediates circadian rhythms in Mammalian olfactory bulb and olfaction. J. Neurosci., 34, 6040-6046.

Murrell, J.R. \& Hunter, D.D. (1999) An olfactory sensory neuron line, odora, properly targets olfactory proteins and responds to odorants. J. Neurosci., 19, 8260-8270.

Negroni, J., Meunier, N., Monnerie, R., Salesse, R., Baly, C., Caillol, M. \& Congar, P. (2012) Neuropeptide y enhances olfactory mucosa responses to odorant in hungry rats. PLoS ONE, 7, e45266.

Ono, D., Honma, S. \& Honma, K. (2015) Circadian PER2::LUC rhythms in the olfactory bulb of freely moving mice depend on the suprachiasmatic nucleus but not on behaviour rhythms. Eur. J. Neurosci, 42, 3128-3137.

Onoda, N. (1992) Odor-induced fos-like immunoreactivity in the rat olfactory bulb. Neurosci. Lett., 137, 157-160.

Raynaud, A., Meunier, N., Acquistapace, A. \& Bombail, V. (2015) Chronic variable stress exposure in male Wistar rats affects the first step of olfactory detection. Behav. Brain Res., 291, 36-45.

Saleh, M., Jurchott, K., Oberland, S., Neuhaus, E.M., Kramer, A. \& Abraham, U. (2015) Genome-wide screen reveals rhythmic regulation of genes involved in odor processing in the olfactory epithelium. J. Biol. Rhythms, 30, 506-518.

Scott, J.W. \& Scott-Johnson, P.E. (2002) The electroolfactogram: a review of its history and uses. Microsc. Res. Tech., 58, 152-160.

Sklar, P.B., Anholt, R.R. \& Snyder, S.H. (1986) The odorant-sensitive adenylate cyclase of olfactory receptor cells. Differential stimulation by distinct classes of odorants. J. Biol. Chem., 261, 15538-15543.

Van Twyver, H. (1969) Sleep patterns of five rodent species. Physiol. Behav., 4, 901-905. 